\title{
Shape-Score verbessert Risikovorhersage von Hüft-Osteoarthritis
}

Gielis WP et al. An automated workflow based on hip shape improves personalized risk prediction for hip osteoarthritis in the CHECK study. Osteoarthritis Cartilage 2020; 28: 62-70

Um die medizinische Betreuung von Patienten mit Hüft-Osteoarthritis (HOA) verbessern und den Krankheitsverlauf positiv beeinflussen zu können, ist es wichtig, eine akkurate Vorhersage über die HOA-Entwicklung treffen zu können. Bisher gibt es kein etabliertes Werkzeug, um die Entwicklung einer 
HOA abschätzen zu können. Gielis et al. entwickelten und validierten ein Prognosetool zur Abschätzung des Risikos für eine zukünftige HOA.

Die Wissenschaftler erstellten ein Vorhersagemodell für HOA, das die automatische Analyse von einfachen Röntgenbildern mithilfe maschinellen Lernens mit klinischen Daten kombiniert. Die Trennschärfe des Modells erwies sich als hoch. Die Forscher schlossen zwischen Oktober 2002 und September 2005 insgesamt $1002 \mathrm{~Pa}$ tienten mit einem Alter zwischen 45-65 Jahren ein, die zur Zeit des Studienbeginns Schmerzen und Steifheit in mindestens einem Knie- oder Hüftgelenk aufwiesen. Die Experten erhoben von den Patienten Daten zum Alter, Geschlecht, BMI, Raucherund Bildungsstatus. Die Wissenschaftler prüften die Patienten auf Hüftschmerzen, wenn diese wegen Schmerzen im Bereich der Leiste, des Gesäßes, im Oberschenkel oder im Knie vorstellig wurden. Die Forscher verwendeten den Western Ontario and McMaster Universities Osteoarthritis Index (WOMAC), um Schmerzen, Steifheit und die körperliche Funktion zu evaluieren. Außerdem sammelten die Forscher Daten zum Schmerzmittelverbrauch, Morgensteifigkeit, vermaßen die aktive Hüftinnenrotation mit einem Goniometer, evaluierten Schmerzen während der Innenrotation und stuften den Schweregrad der Osteoarthritis per Kellgren-Lawrence (KL)-Grad ein. Außerdem fertigten die Experten standardisierte gewichtsbelastende Röntgenaufnahmen des Beckens an. Das Vorhandensein von Gelenkspaltverengungen, Osteophyten, oder einer Verdickung des Oberschenkelknochens wurde auf den Röntgenbildern von geschulten Ärzten beurteilt. Anhand von 75 Landmarken entwickelten die Experten ein vollautomatisches Segmentierungssystem anhand der Röntgenbilder. Anschließend wendeten die Wissenschaftler Algorithmen des maschinellen Lernens an, um einen Shape-Score zu erhalten, der das Risiko für eine zukünftige HOA auf der Grundlage der Gelenkform beschreibt. Um das Vorhersagemodell zu entwickeln, bedienten sich die Experten einer logistischen Regression und fügten Prädiktoren pro Kategorie (demografische Daten, Variablen der klinischen Untersuchung, grundlegende radiologische Parameter und den ShapeScore) hinzu.
$84 \%$ der Patienten waren weiblich, das Durchschnittsalter betrug 56 Jahre, der durchschnittliche BMI 26,3. Von 1044 Patienten zeigten nach 8-jähriger Nachbeobachtung 143 Patienten den KL-Grad 2 oder höher, 42 hatten sich einer Hüft-Totalendoprothese unterzogen. Der Shape-Score erwies sich als signifikanter Prädiktor für eine HOA. Die Trennschärfe der statistischen Modelle verbesserte sich mit jedem neuen hinzugefügten Prädiktor. Das Prädiktionsmodell unter Verwendung der demografischen Daten, den Ergebnissen der körperlichen Untersuchungen und der radiologischen Aufnahmen ergab in Kombination mit dem Shape-Score die stärkste Vorhersagekraft.

\section{FAZIT}

Die Experten entwickelten ein automatisches Shape-Score-Tool, das maschinelle Lernalgorithmen verwendet, um das Risiko für eine HOA auf Grundlage der Hüftform vorherzusagen. Statistische Modelle mit dem entwickelten Shape-Score wiesen im Gegensatz zu jenen ohne Shape-Score eine bessere Trennschärfe auf. Der Shape-Score erwies sich daher als wertvolles Tool, sowohl für die Patientenversorgung als auch für die Forschung, so die Autoren.

Dr. Maddalena Angela Di Lellis, Tübingen 